\title{
Implementation of an FPGA-Based Modem for UAV Surveillance Applications
}

\author{
Michael Rupar \\ John Glancy \\ Naval Research Laboratory \\ Washington, DC \\ And \\ Benjamin Egg \\ Fred Harris \& Associates \\ San Diego, CA
}

\begin{abstract}
Modern Unmanned Aerial Vehicles (UAVs) have the capability of carrying sensors that produce large amounts of data. A high speed wireless data link is required to transfer this data to other systems for processing. This paper presents an FPGA-based implementation of a high data-rate (350 Mbps) QPSK modulator and demodulator to support a high data rate relay from a UAV to a ground station. By using high speed ADCs and DACs in conjunction with state of the art FPGAs it is possible to implement all signal processing functions necessary in a modem. We will present the architecture and methodologies used for both the modulator and demodulator and discuss how these algorithms are implemented in an FPGA.
\end{abstract}

\section{INTRODUCTION}

The threats to our borders and particular to Naval and Coast Guard vessels have continued to escalate with improved technology. The need for more vigilance through improved technology in force and border protection is a necessity. The significant expense of flying manned aerial reconnaissance on a continuous basis drives the requirement for a network of UAVs with advanced communications links back to the command ship or central station to ensure continuous and cost effective surveillance. The video and data transfer rates required for multi-modal persistent surveillance is deemed to be in excess of $1 \mathrm{Gbps}$.

The US Navy dependence on Unmanned Autonomous Vehicles (UAVs) has increased over the past five years, and is expected to do so even more, given the new requirements for force protection and securing of borders. The sensors being flown by these UAVs are growing more and more sophisticated, providing imagery and detection for larger ranges with greater resolution. In addition, the high cost of large UAVs such as the Predator, motivate the deployment of smaller UAVs to enable more frequent and continuous surveillance.

Two such missions envisioned for the leaner, meaner UAVs are for force protection and for border protection. In the first mission, there is a requirement from $\mathrm{CNO}$ to detect potential targets at ranges of $48 \mathrm{~km}$, and to ID those (or other) targets at $24 \mathrm{~km}$. This requirement is applicable on land (in port) as well as at sea. Currently, F$18 \mathrm{~s}$ provide coverage at sea, at great expense. The proposed alternative is to have a number of small UAVs form a surveillance perimeter, with every square $\mathrm{km}$ reexamined in a period less than five minutes. Rather than racing expensive, large, multi-mission UAVs in fast loops around the perimeter, single mission UAVs form a protective ring around the ship or ships, providing the sensor information back to a command point.

The second mission envisions using similarlysized single mission UAVs for Coastal Surveillance of our borders and shipways, where there are long stretches of coastline and many transits, each where there it is necessary to identify small targets. This information is critical to the Coast Guard, the Department of Homeland Security (HLS), as well as the US Navy. Manned fighter aircraft are still the primary option, and it is believed that the lighter ONR/DARPA UAVs could meet this need.

With that increase in sophistication comes an increase in the data link bandwidth required to relay that information back to the command site where it can be assimilated. The capacity for data compression is often overstated. High resolution video, even if compressed successfully to $4 \mathrm{Mbps}$, still exceeds the communications capacity of many existing communications relay systems in the fleet over significant ranges. Given the payload restrictions in size, weight and power (SWAP) of these smaller UAVs, the amount of real-time data assimilation and reduction hardware capable of being carried is further constrained. 
It is therefore believed that to meet the requirement of persistent surveillance, the best solution is to move as much data off the UAV to the command facility in real time with minimal processing and decision making onboard. To do this requires a communication bandwidth in excess of hundreds of megabits per second (Mbps), and in excess of 1 gigabit per second (Gbps) if that information is relayed between UAVs for greater coverage.

Critical to meeting these requirements is to develop a simplex high data rate modem package that is capable of moving traffic at rates faster than $300 \mathrm{Mbps}$ and as fast as 1 Gbps. This paper discusses an FPGA-based implementation of a $350 \mathrm{Mbps}$ simplex QPSK modem.

\section{COMMUNICATIONS SYSTEM SPECIFICATIONS}

The modulator and demodulator that this paper describes are meant to operate in the system specified in Figure 1. In this system the modulator will produce a QPSK signal that is mixed up to a higher frequency for wireless transmission to a tracking earth station. On the ground the signal is mixed back down to an IF, sampled by an ADC and demodulated into the original data stream. The raw data rate of the system is $350 \mathrm{Mbps}$, which occupies a $350 \mathrm{MHz}$ band for QPSK.

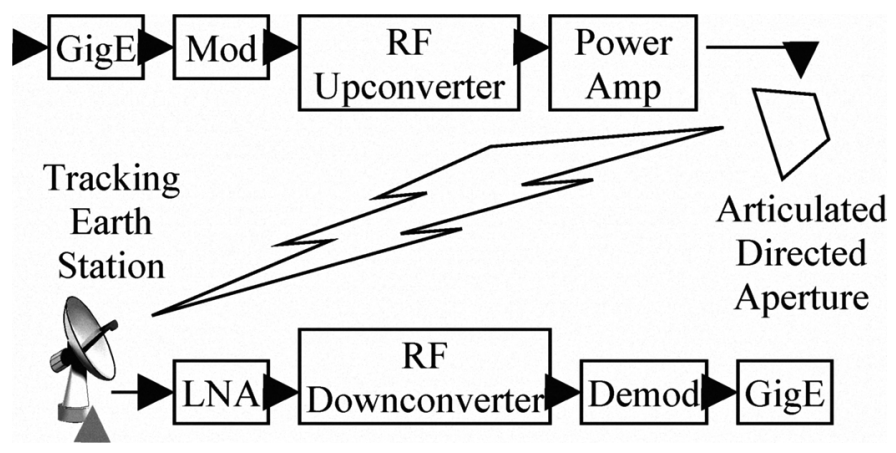

Figure 1. Communication system diagram

\section{HARDWARE PLATFORM}

The hardware platform used to implement the modulator and demodulator was the BDR-1 Basic Digital Radio developed by Fred Harris \& Associates. The BDR1 is a small form factor board that has two Xilinx Virtex-5 SXT50 FPGAs, a Maxim MAX19692 high speed DAC, a Maxim MAX108 high-speed ADC, fixed gain amplifiers, variable gain amplifiers, analog filters, and various high speed I/O interfaces. Figure 2 shows a block diagram of the connections between components on the board [1].

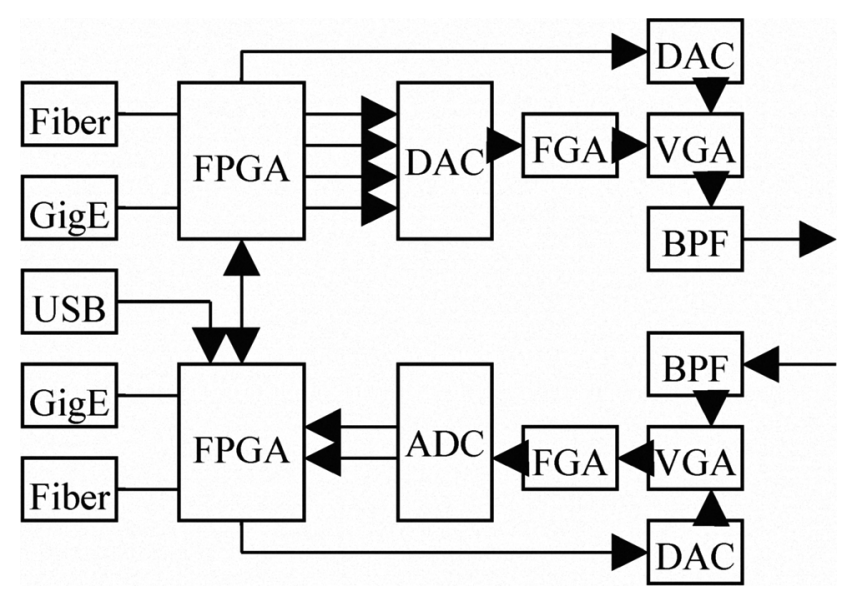

Figure 2. Block diagram of BDR-1 platform

\section{FREQUENCY PLAN}

The RF upconverters being used in our communication system require an L-band IF. On the transmit side we are using a sampling rate of $1.75 \mathrm{GHz}$ and selecting the $2^{\text {nd }}$ Nyquist zone image of the signal with a band pass filter. The center frequency was selected to be $4 / 5$ of the sample rate $(1.4 \mathrm{GHz})$ in order to position the signal in the most linear frequency band of the DAC.

On the receive side we chose to undersample the signal at a sampling rate of $1.4 \mathrm{GHz}$. The center frequency of the signal was chosen to be $3 / 4$ of the sampling rate $(1.05 \mathrm{GHz})$ in order to simplify the digital downconversion process inside the FPGA.

\section{TRANSMITTER IMPLEMENTATION}

The 350 Mbps data rate implies a $175 \mathrm{MHz}$ symbol rate since we are using QPSK modulation. Therefore, it is necessary to interpolate by a factor of 10 to reach the $1.75 \mathrm{GHz}$ sampling frequency of the DAC. The internal logic of the FPGA is not capable of running at the sampling frequency, so some parallelization is required.

Figure 3 shows the implementation used in the FPGA. A polyphase interpolating filter structure is used for the pulse shaping filters in order to reduce the speed of the internal logic. Each filter operates at $175 \mathrm{MHz}$. Since we chose to center the output signal at $4 / 5$ of the sample rate, the sinusoids that are multiplied by the $\mathrm{I}$ and $\mathrm{Q}$ channels repeat every 10 samples. This simplifies the design by allowing us to modulate the filter coefficients instead of the actual data. After the I and Q channels are added together there are 10 parallel data paths. 


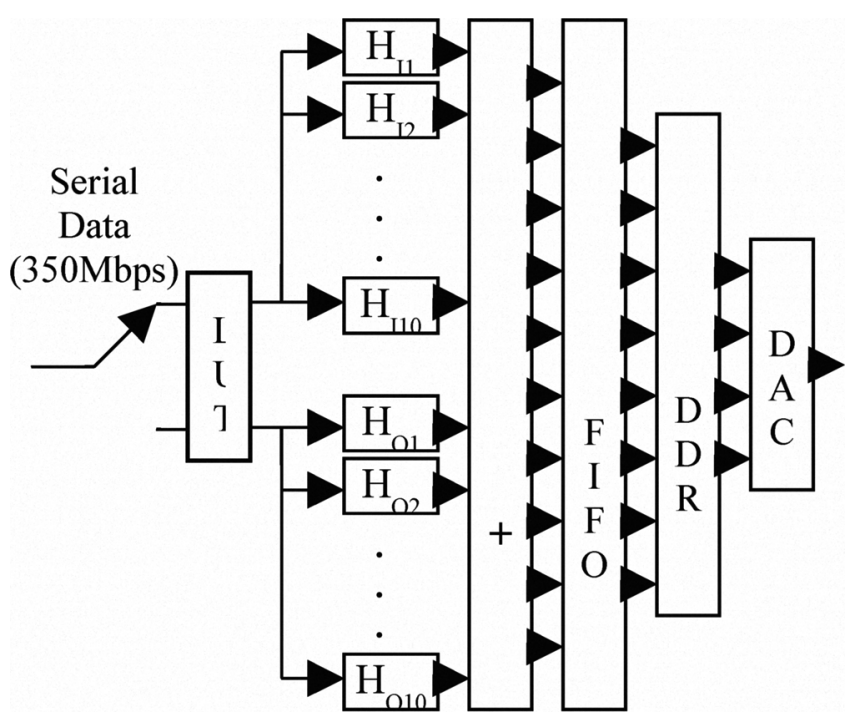

Figure 3. FPGA Implementation of a QPSK modulator

The 10 parallel data paths go into a FIFO structure which is actually an array of 10 asynchronous FIFOs. Additional logic in the FIFO block selects the next 8 samples from the 10 FIFOs to output onto 8 parallel data paths running at $218.75 \mathrm{MHz}$. Figure 4 shows how the outputs of the FIFOs are routed on two consecutive read clock cycles. This pattern continues and repeats every 5 read clock cycles. These 8 paths are sent to DDR output buffers on the FPGA. The DAC internally serializes the 4 DDR channels up to the $1.75 \mathrm{GHz}$ sample rate.

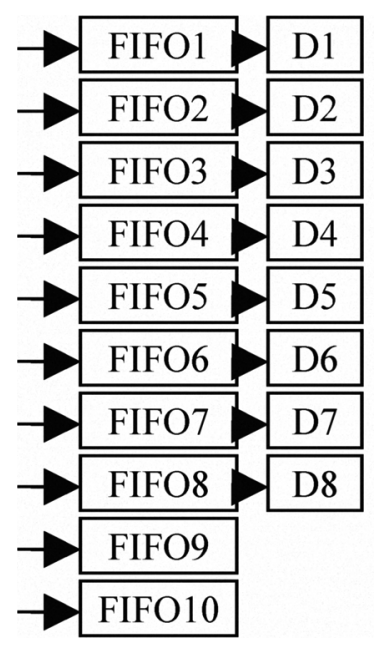

(A)

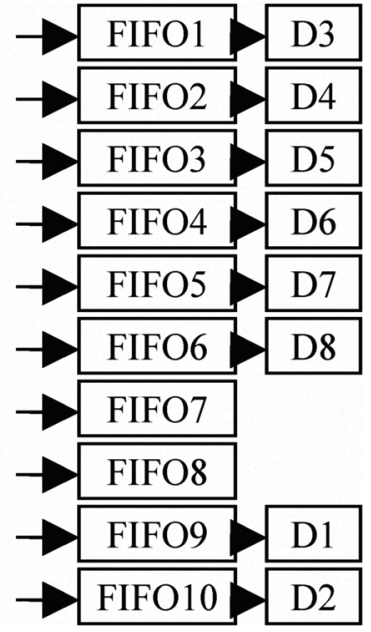

(B)
Figure 4. (A) FIFO structure output at $t=n$. (B) FIFO structure output at $t=n+1$.

\section{RECEIVER IMPLEMENTATION}

The block diagram for a basic QPSK demodulator is shown in Figure 5. The following sections will discuss how each of the components is implemented in the FPGA.

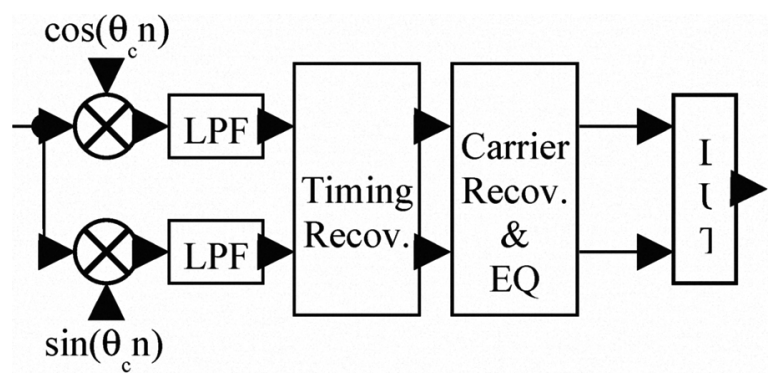

Figure 5. QPSK Demodulator

\section{DIGITAL DOWNCONVERTER}

The digital downconverter (DDC) is simplified due to the center frequency of the received signal being at $3 / 4$ of the sample rate. As shown in figure 6, by implementing the lowpass filter of the I channel as a polyphase decimating filter, it is possible to remove half of the coefficients due to the constant values of the cosine signal used to mix the signal down. Since we are clocking the multipliers at such a high speed, there is very limited reuse of resources. This DDC structure results in a significant savings of multiplier resources. A similar simplification is done to the $\mathrm{Q}$ channel. Following the downconversion, the $\mathrm{I}$ and $\mathrm{Q}$ channels are operating at 2 samples/symbol (350 MHz sample rate).

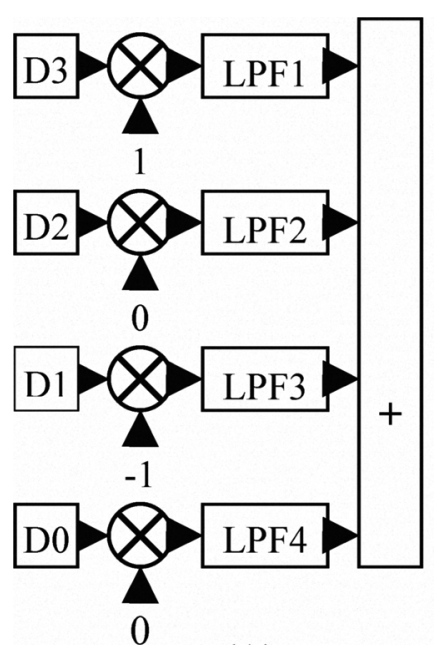

(A)

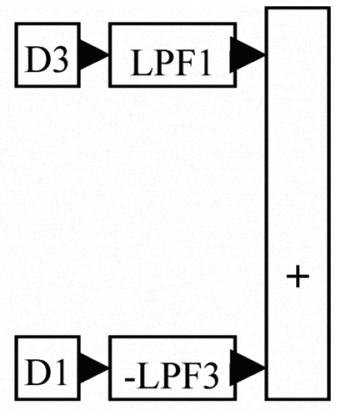

(B)
Figure 6: (A) Basic polyphase digital downconverter.

(B) Simplified polyphase digital downconverter. 


\section{TIMING RECOVERY}

The timing recovery circuit is implemented using a maximum likelihood polyphase matched filter system [3]. Figure 7 shows the block diagram of the timing recovery circuit. This method was chosen due to its quick convergence time. Each filter bank is implemented as a single 12-tap filter with 16 sets of reloadable coefficients stored in memory in order to reduce the number of multipliers used. The timing error is calculated as the product of the matched filter output with the derivative matched filter output. The error is averaged using a firstorder loop filter and then added to an accumulator. The accumulator is used to address the filter bank coefficient memories and select the correct sampling position.

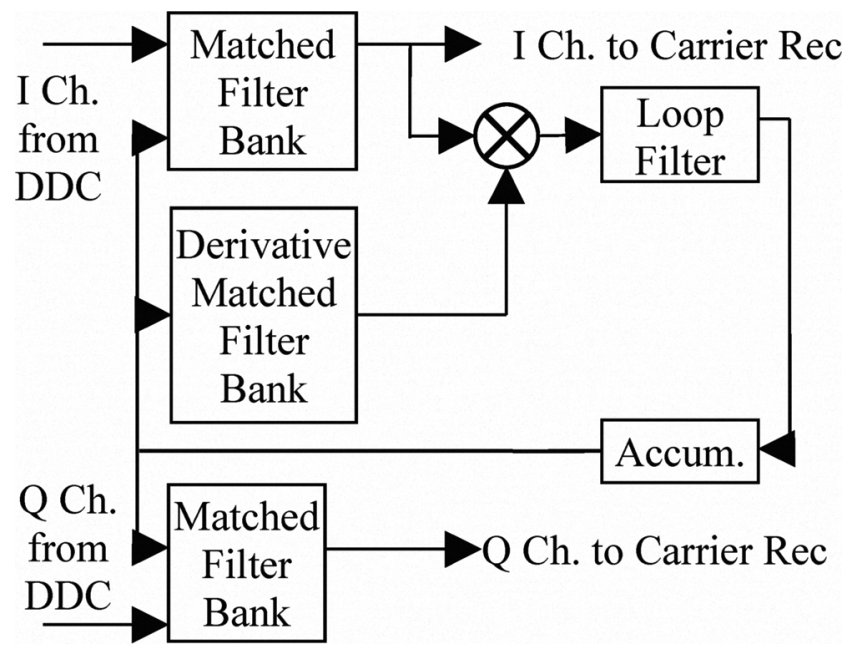

Figure 7: Timing recovery block diagram.

For every symbol period, one sample is a valid data point and the other is a transition point. The top bit of the accumulator selects whether the first or second sample is valid. Accumulator overflow and underflow conditions must be taken into consideration in order to prevent bits from being lost or duplicated in the data stream.

In the overflow case both samples within a symbol period are considered invalid. In the underflow case it is necessary to output two valid samples within a single symbol period. This is done by implementing a second matched filter with constant coefficients. The coefficients are those of the $0^{\text {th }}$ leg of the filter bank. When an underflow occurs the two valid samples for that symbol period are the first sample from the constant coefficient filter and the second sample from the $16^{\text {th }}$ leg of the filter bank.

\section{CARRIER RECOVERY}

In QPSK, an estimate of the phase error can be computed as

$$
e_{p h} \approx Y(n) \operatorname{sign}(X(n))-X(n) \operatorname{sign}(Y(n))
$$

Figure 8 shows the block diagram for the QPSK carrier recovery circuit [5]. Like the timing error, the carrier error is averaged with a first-order loop filter. This value is used to control the frequency of a direct digital synthesizer (DDS) that is used rotate the signal.

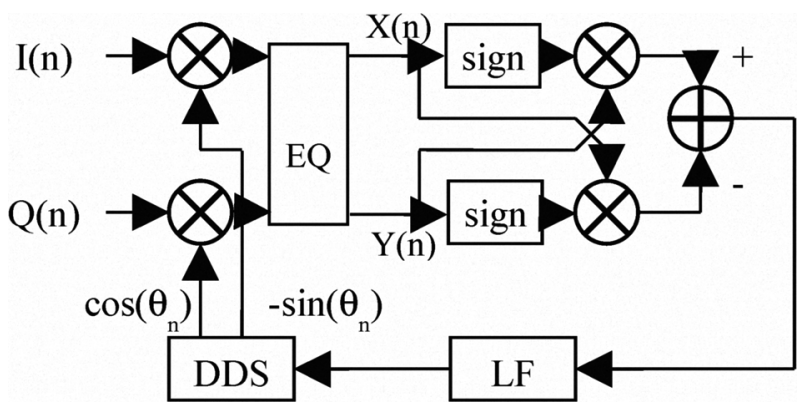

Figure 8: Carrier recovery circuit

\section{EQUALIZER}

The equalization process is essential to minimize the impact of non-ideal RF analog components and dynamic channel conditions. Due to variations over time of both hardware components and channel conditions, the equalization is adaptive, and utilizes a least mean squared (LMS) algorithm to adjust the equalization filter coefficients until the error is minimized.

There are three key parameters we consider to design our adaptive Equalizer. First, since multipath is our largest interferer, we define the maximum time delay (of a multipath reflection) we expect to remove. Second, we specify the worst case relative amplitude of any single multipath reflection (i.e. a reflection from salt water arriving at the antenna $1 / 10$ the magnitude of the primary signal). And third, we specify given 1 and 2, the desired interference cancellation performance we expect (i.e. given a worst case echo $t_{\hat{k}}$ with relative amplitude $\alpha$, we need to attenuate that interferer by Atten $\mathrm{dB}$ ). These parameters define our filter length.

As an example, let's assume the maximum multipath delay is 5 samples $(\mathrm{k}=5)$, the maximum relative amplitude is $1 / 10(\mathrm{a}=.1)$, and the required attenuation of such an interferer is $-40 \mathrm{~dB}$. We determine the length of 
our Equalizer filter in three steps. First we define the echo in the $\mathrm{Z}$ domain in equation 2, and Figure 9:

$$
s(n)=1+\alpha Z^{-k}
$$

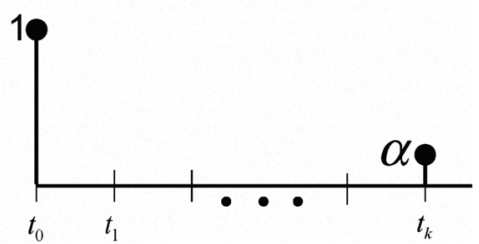

Figure 9: Time representation of main signal and a single reflection of amplitude alpha arriving $\mathrm{k}$ samples later.

The unbounded function that would perfectly remove this multipath component is defined in equation 3 :

$$
\begin{gathered}
H(z)=\frac{1}{1+\alpha Z^{-k}} \\
=1-\alpha Z^{-k}+\alpha^{2} Z^{-2 \hat{k}}-\alpha^{\mathrm{a}} Z^{-\mathrm{z} \hat{k}} \ldots
\end{gathered}
$$

The number of terms we must keep from equation 2 is defined by the desired attenuation of our example, which is $-40 \mathrm{~dB}$ or $\sim .01$ in linear terms. Solving for the desired attenuation as a function of relative amplitude, we use the simple equations in 4.

$$
\begin{gathered}
\text { Atten } n_{\text {linear }}(\alpha)=\alpha^{n} \\
.01=.1^{n} \\
n=2
\end{gathered}
$$

This indicates we keep the terms up to alpha squared.

Now considering the maximum delay path of our example, $Z=5$, we arrive at the filter length that will meet our specifications (5).

$$
\begin{aligned}
& N_{\text {iength }}=k_{\text {deiay }} n_{\text {term }} \\
& \mathrm{N}=5 * 2 \\
& \mathrm{~N}=10 \text { Coefficients }
\end{aligned}
$$

We chose this case as a starting point for our equalizer, so we implemented a complex 10-tap equalizer in the FPGA.

Figure 10 illustrates a basic adaptive FIR filter structure. The parameter $\mu$ is a damping factor which reduces the contribution of each error estimate to slow or speed up the equalizers adaption rate.

It is important to recognize that the equalizer is a complex filter. In other words, it is actually two simultaneous 10-tap FIR filters. Furthermore, since we are operating on complex data, the adaptive equalizer is actually composed of four 10-tap filters. Figure 11 illustrates the 4 paths in the block labeled Complex Equalizer.

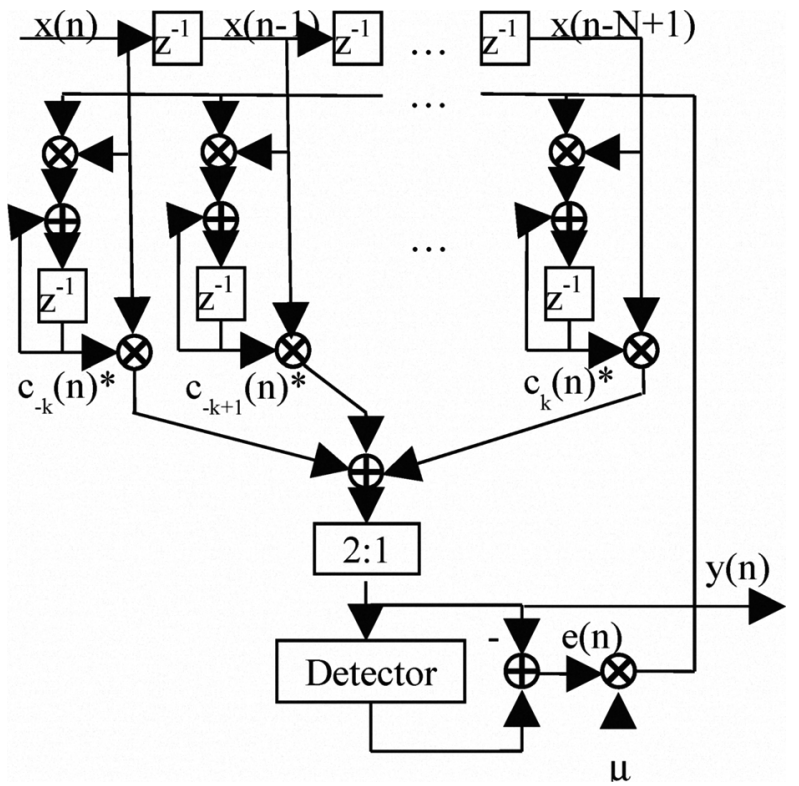

Figure 10. Adaptive FIR Equalizer.

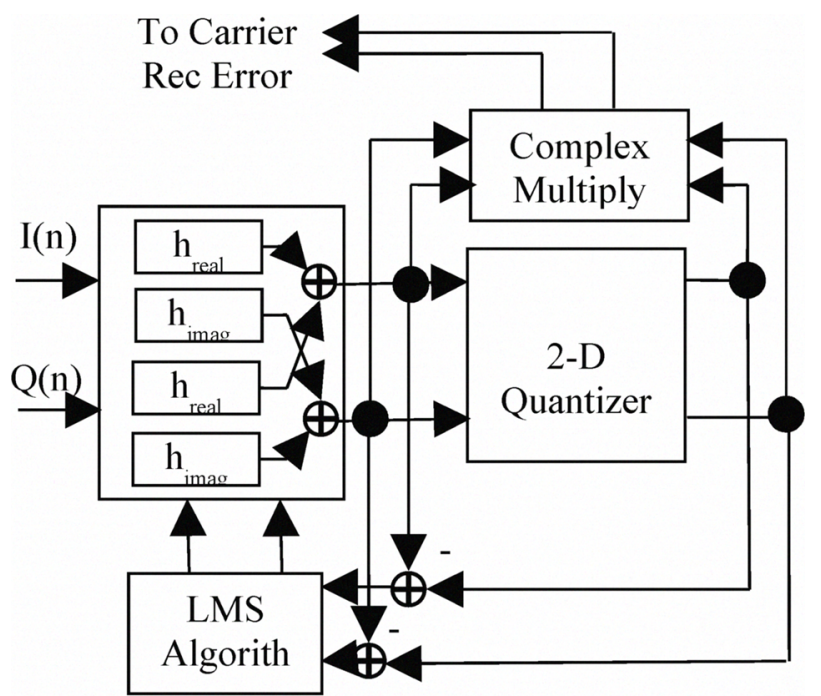

Figure 11: Detailed View of complex signal manipulation. Note that the I \& $Q$ data is operated on by 4 FIR filters.

\section{RESULTS}

Preliminary hardware tests were conducted with the modulator and demodulator in a wired loopback setup using the external RF Upconverter and Downconverter. Figure 12 shows plots of the received constellation and the effect that 
the equalizer has. With the equalizer turned off the timing and carrier recovery loops are able to lock and produce a fairly clean constellation. The constellation points tighten up further with the equalizer turned on. This shows that the equalizer is able to remove some analog distortions that have been introduced into the system. This modem design will be used in September of 2009 to support long range

communications testing, first between ground locations and then from an aircraft to the ground.

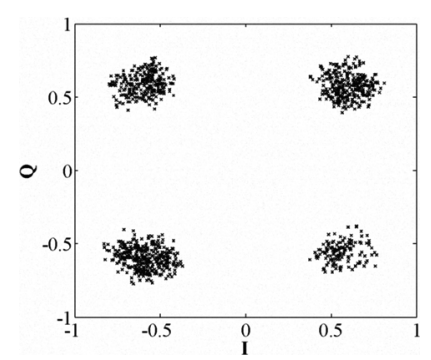

(A)

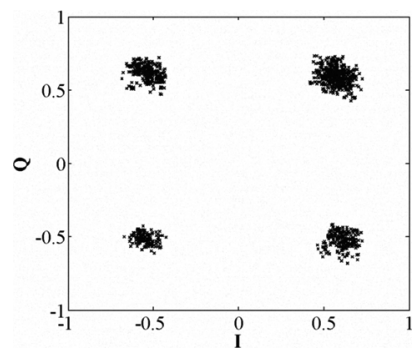

(B)

Figure 12: (A) Carrier recovery output with equalizer off.

(B) Carrier recovery output with equalizer on.

\section{CONCLUSION}

By using the flexible architecture of FPGAs in combination with high-speed DACs and ADCs, it is possible to implement a high-speed modem whose size and power requirements make them an excellent choice for use on UAVs. This particular design does not reach the desired 1 Gbps required for multi-modal persistent surveillance, but it does serve as a step towards higher data-rate designs, and does so with standard commercial interfaces. By using higher order PSK or higher order QAM for the modulation technique it should be possible to increase the overall data rate, and efforts are continuing for a more sophisticated equalizer for better long range performance.

\section{REFERENCES}

\section{[1] Fred Harris \& Associates, "BDR-I User Manual."}

[2] S. Haykin, Communications Systems. New York: John Wiley \& Sons Inc, 2001.

[3] C. Dick, B. Egg, F. Harris, "Architecture and Simulation of Timing Synchronization Circuits for the FPGA Implementation of Narrowband Waveforms," Proceeding of the SDR 06 Technical Conference and Product Exposition. 2006.

[4] L. Litwin, "Matched Filtering and Timing Recovery in Digital Receivers," http://rfdesign.com/mag/ radio_matched_filtering_timing, RFDesign.com, September $200 \overline{1}$. 